\title{
Fiber Coupled Cholesteric Liquid Crystal Laser
}

\author{
M.F. Moreira, I.C.S. Carvalho, \\ Pontifícia Universidade Católica do Rio de Janeiro, Departamento de Física, \\ Cx. Postal 38071, 22452-970, Rio de Janeiro - Brasil \\ L.C.G. Valente, \\ Pontifícia Universidade Católica do Rio de Janeiro, Departamento de Engenharia Mecânica, \\ Cx. Postal 38071, 22452-970, Rio de Janeiro - Brasil \\ P. Palffy-Muhoray, B. Taheri, \\ Liquid Crystal Institute, Kent State University, Kent, OH 44242 - USA \\ and A.F. Muñoz \\ Physics Department, Universidad Autonoma Metropolitana, Mexico City, Mexico
}

Received on 30 November, 2001

\begin{abstract}
Lasing in cholesteric liquid crystals is an exciting new phenomenon. Cholesteric liquid crystals (CLC) are inherently periodic. In their ground state, they form a structure where the optic axis of the uniaxial material twists to form a helix and leads to one dimensional photonic band-gap behavior. If a fluorescent dye with emission matching the reflection band is added to these materials, the distributed feedback properties of the structure can result in laser emission when optically pumped. In this work, we show lasing in a CLC directly coupled to an optical fiber. The laser emission of this device was characterized by pumping the input end of the optical fiber with a nanosecond Nd:YAG $(532 \mathrm{~nm})$ laser.
\end{abstract}

\section{Introduction}

Cholesteric liquid crystals (CLC) are chiral nematics which show a periodic helical structure where the director is perpendicular to the helix axis. The orientation of the director varies linearly with position along the helix axis[1]. The periodic helical structure of cholesteric liquid crystal results in a selective reflection band. In this band, circularly polarized light whose handedness is the same as that of the CLC is totally reflected by a semiinfinite sample. The reflected light has the same handedness as the CLC. The edges of the reflection band are at the free space wavelengths $\lambda_{-}=n_{e} p$ and $\lambda_{+}=n_{o} p$, where $n_{e}$ and $n_{o}$ are the extraordinary and ordinary refractive indices respectively and $p$ is the pitch of the helical structure [2].

If a fluorescent dye, whose fluorescence spectrum overlaps the cholesteric reflection band, is dissolved in the CLC, its fluorescence is suppressed in the reflection band but is enhanced at the band edges. The fluorescence is suppressed due to band gap structure of the CLC. When appropriately chosen CLC-dye mixtures are optically pumped, extremely low threshold lasing can occur at the band edges [3]. The lasing wavelength is determined by the pitch and the refractive index, for example, the wavelength for lasing at the low energy band edge is $\lambda_{-}=n_{e} p$.

Goldberg and Schnur [4] first proposed lasing in CLC systems in 1973. Lasing in dye-doped cholesteric liquid crystal was first demonstrated unambiguously by Kopp et al. [5], and subsequently by Taheri et al. [6] in the visible range. More recently, UV-lasing in pure CLC without the use of dyes was reported by Muñoz et al. [7].

The lasing CLC has opened potential applications as optical sensors and, as we report here, can be directly coupled to optical fibers. Since the wavelength of lasing depends on the material properties of the cholesteric, and since these material properties depend on external factors, the system may be used as a sensor, where the lasing wavelength provides information about the external factors. For example, since the cholesteric pitch $p$ and the refractive indices depend on temperature [2], the lasing frequency can be used as an accurate probe of local temperature. The spectral encoding of information and the possibility of use in conjunction with optical fibers, make this new scheme very attractive.

In this work, we describe the experiment in which the CLC laser is placed on the tip of an optical fiber and pumped by a laser through the fiber. Laser emission 
was observed from the CLC through the fiber, demonstrating the feasibility of using the CLC laser as a fiber sensor.

The use of optical fibers is very advantageous, since it allows the position of the CLC laser, which can be used as a sensor element, to be at distances greater than $10 \mathrm{~km}$ from the reading site. A new configuration for the laser assembly is proposed to efficiently couple the CLC laser to optical fibers.

\section{Experimental Setup}

The schematic of the setup used to couple the CLC laser into the fiber is shown in Fig. 1. The multimode fiber used in this experiment has a $100 \mu \mathrm{m}$ core diameter and a $140 \mu \mathrm{m}$ cladding diameter. The fiber ends were pushed into a Norland UVC Optical Splice connector containing the CLC-dye mixture. The connector consists of a precision alignment guide capable of coupling light from one fiber to the other one. While positioning the fiber ends, we used an optical microscope to adjust the thickness of the cholesteric mixture to approximately $25 \mu \mathrm{m}$.

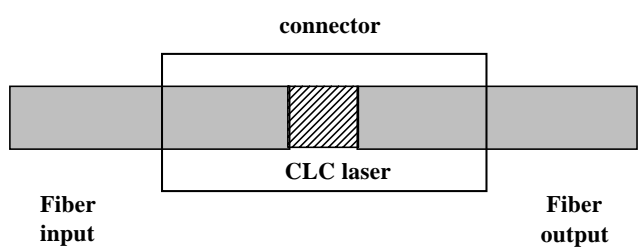

Figure 1. Schematic of the setup used to couple the CLC laser between two multimode fibers.

The sample was a mixture of the CLC BLO61 and the nematic liquid crystal mixture E7. The composition of the sample was $87.2 \mathrm{wt} . \%$ BLO61 and $12.8 \mathrm{wt} . \% \mathrm{E} 7$. The laser dye DCM (4-(dicyanomethylene)-2-methyl-6(4-dimethylamino styryl)-4H-pyran) was then dissolved in the liquid crystal mixture. The dye showed good solubility in the liquid crystal and the dye concentration used was $1.5 \mathrm{wt} . \%$. The final mixture was placed inside of the fiber connector.

The output of a frequency doubled Q-switched $\mathrm{Nd}$ :YAG laser with $7 \mathrm{~ns}$ pulses at wavelength of $\lambda=532$ $n m$ was used as the pump beam in the experiment. The experimental setup is shown in Fig. 2. Two polarizers and a half-wave plate were placed in the optical path of the pump beam to control the pulse energy. This radiation was then coupled to the fiber entrance through a lens $(\mathrm{f}=1 \mathrm{~cm}$ ) and guided through the fiber-CLC-fiber system. Another lens ( $\mathrm{f}=1 \mathrm{~cm}$ ) was used to collect the emitted laser light $\left(\lambda_{2}\right)$ and the pump light $\left(\lambda_{1}\right)$ at the output end of the fiber. The output was focused onto the entrance aperture of the TRIAX 550 spectrometer for characterization of the emission.

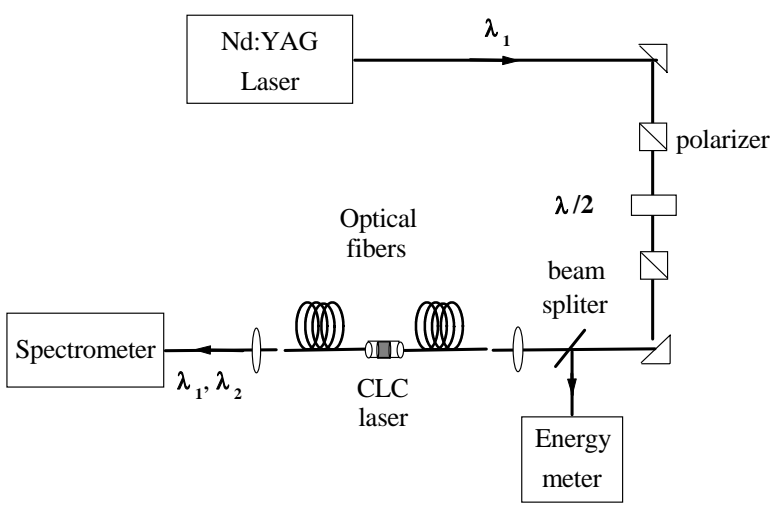

Figure 2. Experimental setup for testing the fiber coupled CLC laser.

\section{Results}

The reflection band for the dye doped CLC was first measured outside the fiber setup using a $23 \mu \mathrm{m}$ cell fabricated using glass coated with ITO and polyimide. The reflection spectrum is shown in Fig. 3. The peak of the fluorescence spectrum $(\sim 580 \mathrm{~nm})$ coincides with the low energy edge of the reflection band of the CLC.

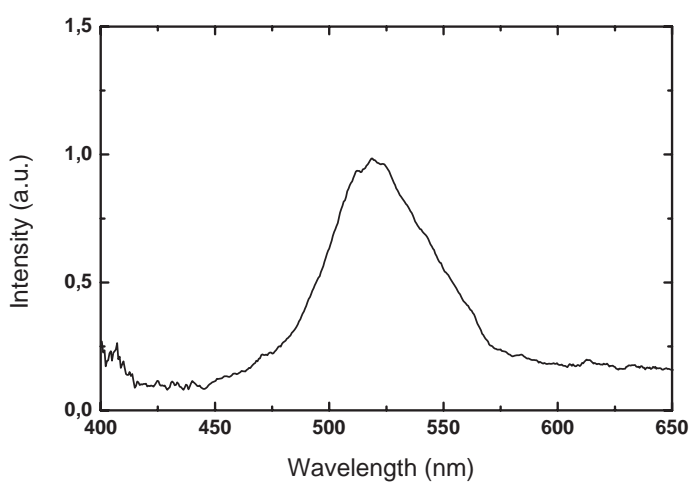

Figure 3. The reflection spectrum of the cholesteric-dye mixture.

The emission spectrum of the CLC laser was measured for pump pulse frequencies of 2,5 and $10 \mathrm{~Hz}$ as shown in Fig. 4. The spectrum presents two emission modes of the CLC laser, characteristic of multi-mode lasing. The bandwidths of first (582.01 nm) and second $(582.65 \mathrm{~nm})$ emission peaks are $1.1 \AA$ and $0.9 \AA$, respectively. 


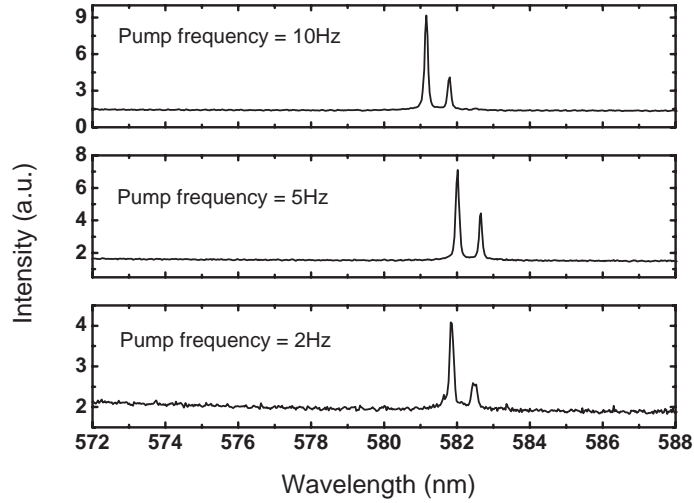

Figure 4. Emission spectrum for the mixture using cells for different laser pump frequencies (a) $2 \mathrm{~Hz}$; (b) $5 \mathrm{~Hz}$ and (c) $10 \mathrm{~Hz}$.

After the characterization of the CLC laser using the cells the sample was placed inside the fiber connector as described above.

Laser emission was detected when the pump pulse from a frequency doubled Nd:YAG laser was launched into the input end of the optical fiber. A typical laser emission spectrum is shown in Fig. 5. The CLC laser emission was at $578.67 \mathrm{~nm}$ with a bandwidth of 1.1 $\AA$. Up to now it had not been possible to stabilize the wavelength of the emission; the fiber CLC laser showed single mode operation, with mode position varying in the $570-600 \mathrm{~nm}$ range with the same bandwidth.

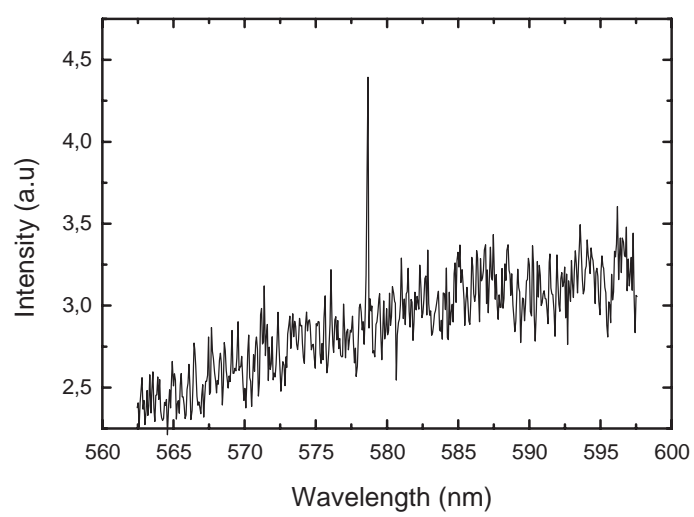

Figure 5. Fiber coupled CLC laser emission.

\section{Conclusion}

We have demonstrated that it is possible to generate laser emission in a CLC laser directly coupled to an optical fiber. The laser emission was unstable, likely due to the lack of well defined liquid crystal alignment at the fiber ends located inside the connector. Achieving such alignment is a difficult task because the fiber is very fragile under the procedures commonly used for glass cells. Other alignment techniques are currently under development. Although the work is at a preliminary stage, the above results strongly suggests the viability of new optical fiber sensors based on the CLC laser.

\section{Acknowledgment}

M.F. Moreira acknowledges studentship from CAPES (Coordenação de Aperfeiçoamento de Pessoal de Nível Superior - Brazil). P. Palffy-Muhoray and B. Taheri acknowledge support from the NSF under ALCOM grant 89-DMR20147, and from the AFOSR under MURI grant F49620-17-1-0014. This work was also supported by an International Collaborative Grant NSF-CNPq (National Science Foundation - USA / Conselho Nacional de Desenvolvimento Científico e Tecnológico -Brazil).

\section{References}

[1] P. Collings, Liquid Crystals - Nature's Delicate Phase of Matter, (Adam Hilger -1990).

[2] P.G.de Gennes and J. Prost, The Physics of Liquid Crystals, (Oxford University Press, Oxford, 1993).

[3] B. Taheri, A.F. Munoz, P. Palffy-Muhoray, and R. Twieg, Mol. Cryst. Liq. Cryst. 358, 73 (2001).

[4] L.S. Goldberg and J.M. Schnur, Tunable internal feedback liquid crystal laser, U.S. patent 3.771 .065 (1973).

[5] V.I Kopp, B. Fan, H.K.M. Vithana and, A.Z. Genack, Opt. Lett. 23, 1709 (1998).

[6] B. Taheri, P. Palffy-Muhoray, and H. Kabir, ALCOM Symposium. Chiral Materials and Applications, Cuyahoga Falls, Feb. 18-19 (1999).

[7] A. Muñoz F., P. Palffy-Muhoray, and B. Taheri, Opt. Lett. 26, 804 (2001). 